\title{
Magnetization Reversal in Granular Nanowires
}

\author{
Hermann Forster, Thomas Schrefl, Rok Dittrich, Dieter Suess, Werner Scholz, Vassilios Tsiantos, Josef Fidler, \\ Kornelius Nielsch, Herbert Hofmeister, Helmut Kronmüller, and Saskia Fischer
}

\begin{abstract}
The switching process of granular Co nanowires is investigated using the finite element method. The wires have a diameter of $55 \mathrm{~nm}$ and a length of $1000 \mathrm{~nm}$. Transmission electron microscopy (TEM) investigations show two different types of hcpstructured grains. For one, the $c$ axis is randomly oriented in a plane perpendicular to the long axis of the wire, and the other has the $c$ axis parallel to the long axis. The numerical results show that finite element micromagnetics can explain the influence of the microstructure in magnetic nanosystems.
\end{abstract}

Index Terms-Micromagnetics, nanowires.

\section{INTRODUCTION}

$\mathbf{P}$ ROGRESS in magnetic storage media is important for the information technology. One possibility to increase the areal density are patterned perpendicular media, where one bit of information corresponds to one single-domain nanosized particle, which is a so-called nanomagnet. For example, an areal density of about $300 \mathrm{GBit} / \mathrm{in}^{2}$ can be achieved by a hexagonal arranged array of nanomagnets with a lattice constant of about $50 \mathrm{~nm}[1]-[3]$.

In this work, an array of Co nanowires is produced based on hexagonally arranged porous-alumina templates [4]. The magnetization is investigated by superconducting quantum interference device (SQUID) magnetometer measurements. A transmission electron microscopy (TEM) image is the starting point for the construction of a finite element model. The simulation is carried out in the framework of micromagnetics.

\section{Finite Element Method in Micromagnetics}

Micromagnetics is a continuum theory for the description of magnetization processes in ferromagnetic materials. The theory starts from the total magnetic Gibbs' free energy [5]

$$
\begin{aligned}
E_{t}=\int\left[A \sum_{i=1}^{3}\left(\nabla \beta_{i}\right)^{2}-K_{u}(\mathbf{u} \cdot \beta)^{2}\right. & \\
& \left.-\frac{1}{2} \mathbf{J} \cdot \mathbf{H}_{d}-\mathbf{J} \cdot \mathbf{H}_{\mathrm{tot}}\right] d V
\end{aligned}
$$

where $A$ is the exchange constant, $\beta_{i}$ denotes the direction cosines of the magnetic polarization vector, and

\footnotetext{
Manuscript received February 14, 2002; revised May 19, 2002. This work was supported by the Austrian Science fund (Y132-PHY).

H. Forster, T. Schrefl, R. Dittrich, D. Suess, W. Scholz, V. Tsiantos, and J. Fidler are with Vienna University of Technology, Vienna, Austria (e-mail: hermann.forster@tuwien.ac.at).

K. Nielsch and H. Hofmeister are with Max-Planck-Institute of Microstructure Physics, Halle, Germany.

H. Kronmüller is with Max-Planck-Institute of Metal Research, Stuttgart, Germany.

S. Fischer is with Department of Electrical Engineering, Ruhr University Bochum, Bochum, Germany.

Digital Object Identifier 10.1109/TMAG.2002.801958
}

$\mathbf{J}=\left(\beta_{1}, \beta_{2}, \beta_{3}\right) J_{s} . K_{u}$ and $\mathbf{u}$ are the magneto-crystalline anisotropy constant and the anisotropy direction. $\mathbf{H}_{\text {tot }}$ is the total field acting on one wire. The demagnetizing field $\mathbf{H}_{d}$ follows from a magnetic scalar potential. The magnetic scalar potential solves the magnetostatic boundary value problem and can be effectively computed using a hybrid finite element/boundary element method [6]. Since $A, J_{s}$, and $K_{u}$ are a function of space, we have the possibility to describe granular structures.

The actual path of the system toward a local minimum is obtained from the time integration of the Gilbert equation of motion [7]

$$
\frac{\partial \mathbf{J}}{\partial t}=-|\gamma| \mathbf{J} \times \mathbf{H}_{\mathrm{eff}}+\frac{\alpha}{J_{s}} \mathbf{J} \times \frac{\partial \mathbf{J}}{\partial \mathbf{t}} .
$$

The effective field $\mathbf{H}_{\text {eff }}$, which provides the torque acting on the magnetization, is the negative variational derivative of the magnetic Gibbs' free energy $\mathbf{H}_{\mathrm{eff}}=-\delta \mathbf{E}_{t} / \delta \mathbf{J}$. The first term describes the gyromagnetic precession, where $\gamma$ is the gyromagnetic ratio. The second term describes the damping, which is characterized by the Gilbert damping constant $\alpha$. In equilibrium, the torque $\mathbf{J} \times \mathbf{H}_{\text {eff }}$ vanishes, and the magnetic polarization is at rest.

To solve the partial differential (2) for the whole sample, we use the finite element method. Therefore, we have to divide the magnetic sample into tetrahedral finite elements. Within each element, the direction cosines $\beta_{i}$ are interpolated by a linear function. The time integration is performed using a preconditioned backward differentiation method (BDF) [8].

\section{SAMPLE GEOMETRY}

Co nanowires were grown in highly ordered anodic alumina templates using electrodeposition. This technique yields completely metal-filled alumina membranes. The nanowires are arranged in a hexagonal lattice. The diameter of one nanowire is $55 \mathrm{~nm}$, and the lattice spacing is $100 \mathrm{~nm}$. The typical length is $1000 \mathrm{~nm}$.

Fig. 1 shows a TEM image of two Co nanowires. The investigations show that there are two types of grains, both with hcp structure, where the $c$ axis is the magnetocrystalline easy direction. The first type has the $c$ axis randomly oriented in a plane perpendicular to the long axis of the wire. The typical length of these grains is $100-250 \mathrm{~nm}$. Grains of the second type have a length of less than $100 \mathrm{~nm}$, and the $c$-axes are parallel to the long axis. The first kind of grains has a total volume fraction of the Co nanowire of $70 \%-90 \%$.

The simulation of an array of nanowires would exceed computational power. Therefore, we simply model a finite element model one nanowire. The influence of the other nanowires due to 


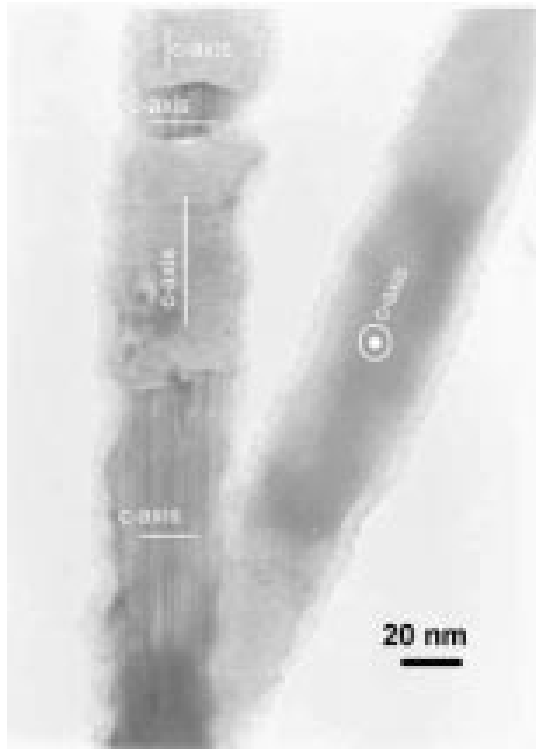

Fig. 1. TEM image of various Co-nanowires. The $c$ axis of the hcp structure is indicated

the stray field is taken into account by a demagnetization factor. Our model of one nanowire consists of seven Co-grains. The material parameters are $J_{s}=1.76 \mathrm{~T}, A=1.3 \times 10^{-11} \mathrm{~J} / \mathrm{m}$, and $K_{u}=4.5 \times 10^{5} \mathrm{~J} / \mathrm{m}^{3}$ [9]. The total length of the sample is 1000 $\mathrm{nm}$, and the diameter is $55 \mathrm{~nm}$. The simulations showed that the results are insensitive to a reduction of the exchange coupling between the grains. Fig. 4 shows the geometry of our model. The smaller grains have a magnetocrysalline easy direction parallel to the long axis, instead of the bigger grains, where the easy axes are randomly distributed in a plane perpendicular to the long axis, which have a total volume fraction of $75 \%$. The results are independent of the mesh size $h$, if $h \leq 10 \mathrm{~nm}$. Since we are interested in equilibrium properties, it is enough to use an element size of 10 $\mathrm{nm}$, which results in 4387 nodes and 16960 elements.

\section{RESULTS}

\section{A. Experimental Results}

The magnetic properties of Co-filled nanopore arrays are carefully investigated by SQUID magnetometer measurements. The external field is applied parallel and perpendicular to the long axis. The hysteresis curves are measured parallel to the external field. Fig. 2 shows the result. Both orientations show only a small hysteretic behavior, resulting in small remanence $\left(J_{R} / J_{S} \leq 11 \%\right)$ and coercive field $\left(H_{C}^{\|} \approx 40 \mathrm{kA} / \mathrm{m}\right.$, $\left.H_{C}^{\perp} \approx 20 \mathrm{kA} / \mathrm{m}\right)$. The results suggest that the sample is magnetically hard parallel to the long axis. This unusual behavior can be explained by the specific arrangement of the magnetocrystalline anisotropy axes and the global demagnetizing factor of the two-dimensional (2-D) array of nanowires.

\section{B. Simulation}

For the simulation, we use the model described above. The external field is applied parallel and perpendicular to the long axis. Initial point is the Co nanowire that is totally saturated parallel to the long axis. Then, the external field is instantaneously

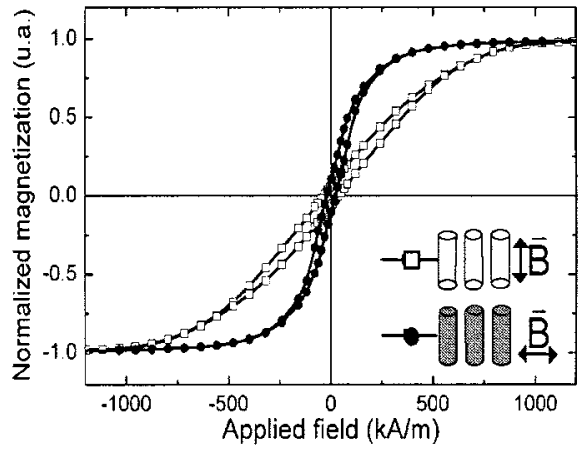

Fig. 2. Measured hysteresis curves for applied field parallel (open diamonds) and perpendicular (closed circles) to the long axis of the wires.

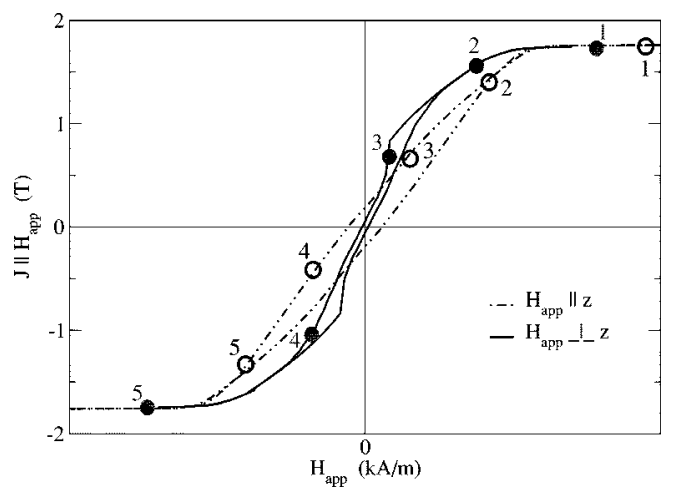

Fig. 3. Hysteresis curves for applied field parallel (dashed lines) and perpendicular (full lines) to the long axis. Numbered points correspond to snapshots in Figs. 4 and 5.

applied with a strength of $1400 \mathrm{kA} / \mathrm{m}$. For each applied field value, we integrate (2) until the equilibrium state is reached, and then, the external field is reduced by steps of $28 \mathrm{kA} / \mathrm{m}$. After the simulation of the total hysteresis curves, we have to consider that we just simulate one nanowire, whereas the experimental measurement is the result of the interaction within a 2-D array of nanowires.

In an experimental setup, the total field acting on one wire is the sum of the applied field and the demagnetizing field of the 2-D array

$$
H_{\text {tot }}=H_{\text {app }}+H_{\text {demag }}
$$

where $H_{\text {demag }}=-N J / \mu_{0}$. To compare the simulation result with the experiment, we have to plot $H_{\text {app }}$ versus $J$, which is $\left(H_{\text {tot }}+N J / \mu_{0}\right)$ versus $J$. The demagnetizing factor $N$ depends on the direction. In our case, we have to distinguish between directions parallel and perpendicular to the plane of the 2-D array of nanowires. The demagnetizing factor perpendicular to the film plane is 1 for a fully dense infinitely extended thin film. Taking into account a packing density of 30\%, which follows from the wire diameter and the spacing, the demagnetizing factor is reduced to 0.3 , assuming linear scaling. Numerical investigations of the influence of the packing factor on the interaction field and the coercivity [10] show that the linear scaling does not hold for a small packing factor. Our simulations show a qualitative agreement with the experiment for a demagnetizing factor greater than 0.5 . 


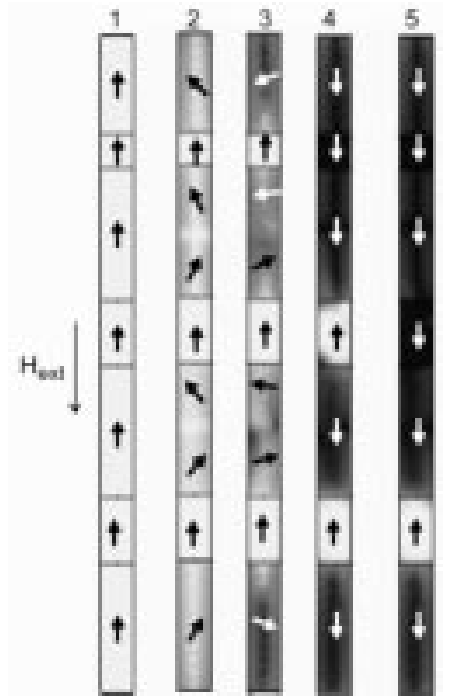

Fig. 4. Snapshots of the magnetization reversal according to the open circles in Fig. 3. The external field is applied parallel to the long axis. The greyscale represents the magnetization parallel to the long axis.

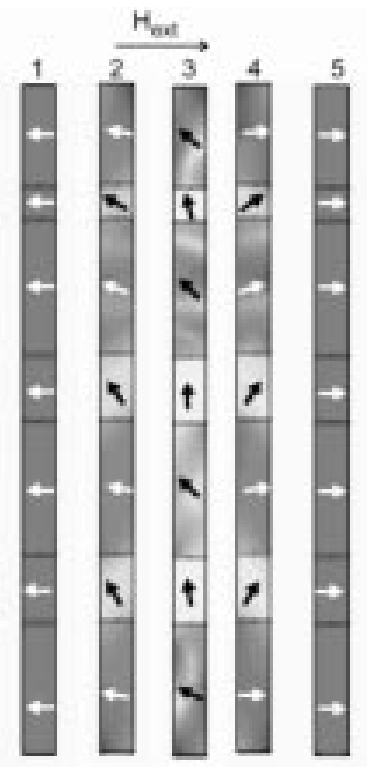

Fig. 5. Snapshots of the magnetization reversal according to the full circles in Fig. 3. The external field is applied perpendicular to the long axis. The greyscale represents the magnetization parallel to the long axis.

The hysteresis curves for $N=0.6$ can be seen in Fig. 3. Obviously, there is a good qualitative agreement with the exper- imental result of Fig. 2. The nanowire shows just a small hysteretic behavior. The values for the coercive fields are by a factor 2 bigger than the experimental values. Figs. 4 and 5 show snapshots of the magnetization distribution in the nanowire during the magnetization reversal. The greyscale represents the magnetization parallel to the long axis. For the perpendicular case, the magnetization starts to turn in the smaller grains, which have a magnetocrystalline easy direction parallel to the long axis. As the external field is sufficiently decreased as well, the magnetization in the bigger grains with a magnetocrystalline easy direction perpendicular to the long axis starts to rotate, but in these grains, the reversal is finished earlier, due to the fact that the external field has to be strong to rotate the magnetization out of the magnetocrystalline anisotropy direction in the smaller grains. Contrary in the parallel case, the reversal starts in the bigger grains.

We also performed simulations neglecting the granular structure of the Co nanowire. This means that the nanowire is just one single crystal with uniaxial anisotropy that is either parallel or perpendicular to the long axis. The simulations clearly show that we do have to take into account the granular structure to achieve a good qualitative agreement with the experiment.

\section{REFERENCES}

[1] K. Nielsch, R. B. Wehrspohn, S. F. Fischer, H. Kronmüller, J. Barthel, J. Kirschner, and U. Gösele, "Magnetic properties of 100 nm-period Nickel nanowire arrays obtained from ordered porous-alumina templates," in Proc. Mat. Res. Soc. Symp., vol. 636, 2001, pp. D1.9.1-D1.9.6.

[2] R. Hertel, "Micromagnetic simulations of magnetostatically coupled Nickel nanowires," J. Appl. Phys., vol. 90, pp. 5752-5758, 2001.

[3] K. Nielsch, R. Hertel, R. B. Wehrspohn, J. Barthel, J. Kirschner, U. Gösele, S. F. Fischer, and H. Kronmüller, "Switching behavior of single nanowires inside dense Nickel nanowire arrays," IEEE Trans. Magn., to be published.

[4] K. Nielsch, R. B. Wehrspohn, J. Barthel, J. Kirschner, U. Gösele, S. F. Fischer, and H. Kronmüller, "Hexagonally ordered $100 \mathrm{~nm}$ period Nickel nanowire arrays," Appl. Phys. Lett., vol. 79, pp. 1360-1362, 2001

[5] W. F. Brown, Micromagnetics. New York: Wiley, 1963.

[6] D. R. Fredkin and T. R. Koehler, "Hybrid method for computing demagnetizing fields," IEEE Trans. Magn., vol. 26, pp. 415-417, Apr. 1990.

[7] T. L. Gilbert, "A lagrangian formulation of gyromagnetic equation of the magnetization field," Phys. Rev., vol. 100, p. 1243, 1955.

[8] D. Suess, V. Tsiantos, T. Schrefl, J. Fidler, W. Scholz, H. Forster, and R. Dittrich, "Time resolved micromagnetics using a preconditioned finite element method," J. Magn. Magn. Mater., 2002, to be published.

[9] W. Yang, D. N. Lambeth, and D. E. Laughlin, "Dependence of Co anisotropy constants on temperature, processing and underlayer," $J$. Appl. Phys., vol. 87, pp. 6884-6886, 2000.

[10] C. R. Chang and J. S. Yang, "Influence of packing factor on coercivity of particle arrays," J. Magn. Magn. Mater., vol. 155, pp. 92-94, 1996. 